\title{
Malignant Melanoma Arising of Gland Pigmentary Disorders
}

\author{
Vitorino Modesto dos Santos* and Lister Arruda Modesto dos Santos
}

Armed Forces Hospital, Estrada do Contorno do Bosque, Brazil

*Corresponding author: Prof. Dr. Vitorino Modesto dos Santos, Armed Forces Hospital, Estrada do Contorno do Bosque, Cruzeiro Novo, CEP 70658-900, Brasília-DF, Brazil, Tel: \#55-61-39662103, Fax: \#55-61-32331599

\begin{abstract}
Malignant melanoma represents up to $5 \%$ of cutaneous cancers, but up to $75 \%$ of the cases have fatal outcomes. The early diagnosis and prompt treatment can reduce the impressive mortality rate of this ominous malignancy. Both amelanotic and melanotic melanoma may be associated with delayed clinical suspicion and late diagnosis, harmful occurrence that may be related to lack of awareness of health care workers or unusual site of the tumors. The manuscripts herein commented described exceeding rare presentations of this challenging malignant tumor. The purpose is to enhance the suspicion index of primary health care workers about uncommon clinical features.
\end{abstract}

\section{Keywords}

Diagnosis, Glands, Malignant melanoma

We have read with special interest the article published in this Journal by Ewing $E$, et al. about a 75-yearold man with a primary malignant melanoma detected in area of nasal glands containing melanocytes [1]. Primary intranasal site of this malignancy is considered an exceeding rare condition ( $1 \%$ of all locations); moreover, unspecific symptoms mimicking common upper airway affections may propitiate diagnostic delay [1]. In the commented case, the occurrence of bleeding called attention to a more severe disorder deserving specialized evaluation. The biopsy of nasal mass established the correct diagnosis of spindle cell melanoma. Differing from the majority of previous literature data, pigmented cells were found in the seromucous glands. Although melanin pigmentation may occur in nasal glands and dendritic cells, malignant melanoma was not previously described with origin in nasal seromucous glands containing melanocytes and melan pigment [1]. The authors found melanosis with HMB-45 and melan-A immunoreactivity, and positive in Fontana-Masson. Their novel described finding was melanin with a pattern similar to that of melanotic oncocytic metaplasia, in which dendritic melanocytes appear S-100 positive but HMB- 45 negative by immunohistochemistry studies [1]. Additionally, to the highly significant contribution to the knowledge about this very ominous malignancy, they emphasized the role of persistent nasal obstruction, epistaxis, or hemoptysis as suspicion index of malignancy. The enhanced awareness of general practitioners and primary health care workers will improve early diagnosis.

In the scenery of malignant melanoma in exocrine glands, we add short comments about diagnostic challenges between primary or secondary parotid amelanotic malignant melanoma in a Brazilian woman [2]. Routine histopathology and immunohistochemistry of the excised gland revealed a cystic metastatic amelanotic melanoma within the parotid, and primary site in the scalp; as usual, it had longstanding unsuspected evolution. The authors highlighted the positivity for S-100 protein and Melan-A in immunohistochemistry evaluation of surgical specimens from intraparotid and supraclavicular lymph nodes, consistent with malignant melanoma. Diagnostic pitfalls involving this type of melanoma are more common and late diagnosis usually increases the morbidity and mortality; a main differential hypothesis is metastasis to lymph node within the parotid gland [2]. The authors described the dermoscopy evaluation of the primary melanoma on the scalp, which was removed. Similarly, to the very illustrative review done by authors of the rare primary intranasal malignant melanoma, Brazilian authors shortly reviewed data about the rare intraparotid cystic metastasis of amelanotic melanoma [2]. 
One should also emphasize data from fine needle aspiration biopsy (FNAB) in 184 metastatic tumors of salivary glands, in six tertiary medical centers of the United States and Europe, described by Wang, et al. [3]. Among the studied patients, metastatic malignant melanoma was the second most common malignancy with 67 (36.4\%) cases; 52 (77.6\%) originated from head and neck, ten (14.9\%) from other sites, and five (7.5\%) were limited to the intraparotid lymph nodes. Twenty-one (31.3\%) of all metastases had melanin, whereas $7.4 \%$ of them presented spindle cell components. Clinical and pathologic features were: Male gender (83.6\%); mean age 66 (25-88) years; known diagnosis before FNAB (92.5\%); and mean time of 14.8 months between FNAB and known diagnosis. One patient had metastasis 219 months after the diagnosis of the primary site of melanoma. Because of the rarity, publications on metastatic tumors to salivary glands are in small series or case reports [3].

The main purpose of the manuscripts herein commented is to valorize the accurate physical inspection of the whole body in order to enhance the rate of early di- agnosis related to cutaneous and mucosal malignancies. Moreover, the authors concluded that FNAB can be useful for the detection and diagnosis of these conditions.

\section{Financial Disclosure}

There was no grant support for this study.

\section{Disclosure of Potential Conflicts of Interest}

The authors had full freedom of manuscript preparation and there were no potential conflicts of interest.

\section{References}

1. Ewing E, Jorgensen JB, Erhardt BF, Layfield LJ (2017) Malignant melanoma arising in association with sinonasal melanosis: a case report and review of the literature. Int $J$ Pathol Clin Res 3: 058.

2. Santos VM, Gondim Neto Mda C, de Melo TR, Motta IM (2016) Parotid cystic lesion in amelanotic malignant melanoma. J Coll Physicians Surg Pak 26: 781-783.

3. Wang H, Hoda RS, Faquin W, Rossi ED, Hotchandani N, et al. (2017) FNA biopsy of secondary nonlymphomatous malignancies in salivary glands: A multi-institutional study of 184 cases. Cancer Cytopathol 125: 91-103. 